\title{
A Knowledge Modeling Method for Computer Graphics Design \& Production Based on Ontology
}

\author{
Tong Chen ${ }^{1}$, Beibei Fan ${ }^{* 1}$, Dongping Chen ${ }^{2}$ and Cong $\mathrm{Li}^{1}$ \\ ${ }^{1}$ Shanghai University, School of Mechatronic Engineering and Automation, 200072. \\ ${ }^{2}$ AVIC Commercial Aircraft Engine Co., Ltd, Shanghai 200241, China. \\ *Corresponding author: fanbeibei@shu.edu.cn
}

\begin{abstract}
As one of the most critical stages of CG (Computer Graphics) industry, CG design \& production needs the support of professional knowledge and practice experience of multidisciplinary. With the outstanding performance in knowledge sharing, integration and reuse, knowledge modeling could increase greatly the efficiency, reduce the cost and avoid repeated error in CG design \& production. However, knowledge modeling of CG design \& production differs greatly from those of other fields. On the one hand, it is similar to physical product design, which involves great deal of tacit knowledge such as modeling skills, reasoning knowledge and so on. On the other hand, as film, CG design \& production needs a lot of unstructured description information. The heterogeneity between physical product and film makes knowledge modeling more complicated. Thus a systematic knowledge modelling method based on Ontology is proposed to aid CG design \& production in this paper. CG animation knowledge is capture and organized from viewpoint of three aspects: requirements and design and production. The knowledge are categorized into static and dynamic knowledge, and Ontology is adopted to construct a hierarchic model to organize the knowledge, so as to offer a uniform communication semantic foundations for designers from different fields. Based on animation script, the CG design task model is proposed to drive the organization and management of different knowledge involved in CG design \& production. Finally, we apply this method in the knowledge modeling of naked-eye animation design and production to illustrate effectiveness of this method.
\end{abstract}

Key words. CG; Knowledge Modeling, Ontology, OWL, Static Knowledge, Dynamic Knowledge.

\section{Introduction}

CG (Computer Graphics) animation is a kind of film designed and produced mainly by computer. CG animation is an important media product, which is of great need in film industry, industrial products propaganda, education and some other fields. As one of the most critical stages of CG industry, CG design \& production involves script design, 3D modeling, scene rendering, road planning and so on. Characterized by high density of knowledge and innovation, CG design \& production needs the support of professional knowledge and practice experience of multidisciplinary. CG design $\&$ production is the process to transform the customer requirements into CG animation by related personnel (shown in Figure 1). A great deal of knowledge would be generated during this process, such as story, story board, scripts, modelling skills, parameters setting methods and so on. 


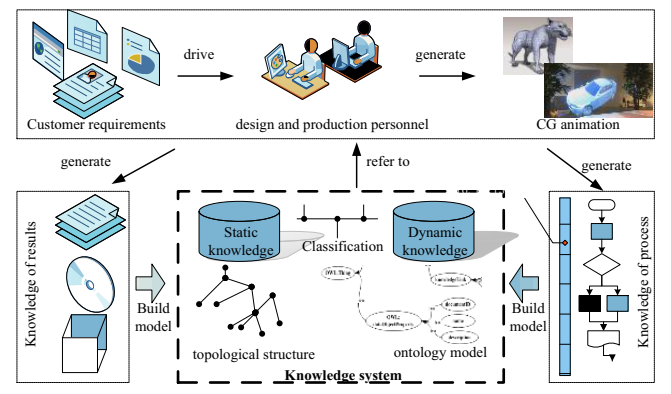

Figure 1. The framework of CG design \& production process knowledge modeling

With the outstanding performance in knowledge sharing, integration and reuse, knowledge modeling could increase greatly the efficiency, reduce the cost and avoid repeated error in CG design \& production. The Ontology technique is an important tool in knowledge engineering, which could support knowledge sharing, integrating and reusing on the level of semantic, and describe various kinds of phenomenon, facts and theoretical knowledge[1]. However, knowledge modeling of CG design \& production differs greatly from those of other fields. On the one hand, it is similar to physical product design, which involves great deal of tacit knowledge such as modeling skills, reasoning knowledge and so on. On the other hand, as film, CG design \& production needs a lot of unstructured description information. The heterogeneity between physical product and film makes knowledge modeling more complicated. Thus, this article aims to propose an integrating knowledge modelling method to aid CG design \& production. The following part of this paper is as follows: related works on Ontology are presented in Part 2. CG design \& production process is stated, and the knowledge generated during this process is analyzed in Part 3. The Ontology modelling process is illustrated in Part 4. A case of 3D film design \& production is illustrated in Part 5. Part 6 concludes the whole paper.

\section{Related works}

Ontology is a philosophy terminology referred to the ultimate physical phenomenon which states philosophical theories. In the realm of knowledge engineering, there are no universal definition of Ontology. The most famous one is defined by Gruber-- "Ontology is a description of the concept of shared conceptualization"[2]. Ontology is studied and applied in many aspects, such as geographical area [3], biological and medical field[4-5], assembling process domain[6-7], service area[1] and so on.

The work of movie Ontology building is really great in progress. Some famous knowledge databases such as LinkedMdb, Freebase and so on, have been built on the open datacloud[8]. Wang[8] proposed a method to construct Bilingual Movie Knowledge Graph. However, these knowledge databases focus on film information about actors, audience, production companies and so on. Little knowledge about the design \& production is involved.

Most Ontology researches about design \& production were carried on tangible products[9-11]. Zhou et al. [9]proposed a modeling method of Diesel Ontology Model for Multidisciplinary Optimization Process, and solved the problems that sematic of information model was simple and information could not be inherited shared. However, as film, CG design \& production needs a lot of unstructured description information. Thus the method of tangible products design \& production knowledge building could not be used in CG directly.

\section{CG design \& production process analysis}

CG design \& production is the process to transform the customer requirements into CG animation by related personnel. A great deal of knowledge would be generated during this process. The detailed procedures are outlined in Figure 2. Story, story board, checking video, script and other kind of knowledge are generated in the design stage, while 3D model, camera layout, lighting layout, background materials are generated in the production stage. Besides, modelling skills, 
parameters setting methods and other tacit knowledge will be generated during the whole process. The above mentioned knowledge constitutes the knowledge system of CG design \& production process.

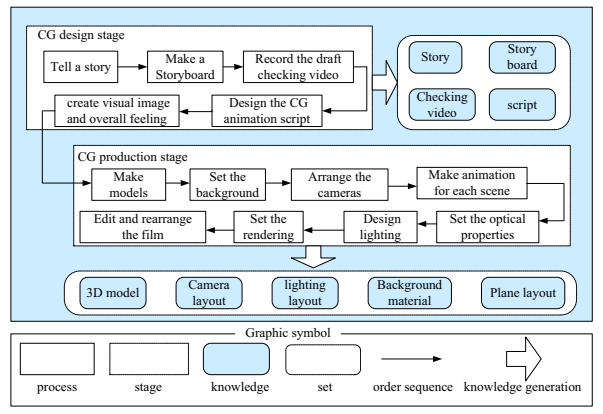

Figure 2. The knowledge generated within CG design \& production process

In order to share information, the knowledge are classified into static knowledge and dynamic knowledge. Static knowledge could be referred to directly in other fields, while dynamic knowledge could be used in CG design \& production process only. The upper part of Figure 3 shows the knowledge system classification of CG design \& production process.

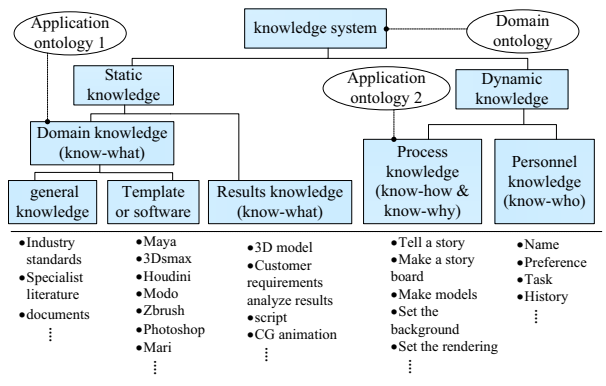

Figure 3. The knowledge system classification of CG design \& production process

The main tasks of knowledge modeling in this article are to build the domain Ontology model and application Ontology model for each kind of knowledge appeared in Figure 2. The domain Ontology of CG design \& production process is the base model for the classified knowledge. Due to limited space, only the domain Ontology model and two application ontologies (one in static knowledge sub-class and another in dynamic knowledge shown in Figure 3) are demonstrated in the following part.

\section{Ontology modelling for CG design \& production process}

The domain Ontology of CG design \& production process is the base model for the classified knowledge. Ontology model could be constructed by two methods - manual modeling and computer modeling. One the one hand, manual modeling is excellent in recognizing important concepts by experts due to the comprehensive property of human, while prone to omitting some details. On the other hand, computer modeling performed well in dealing with mass data, but can't summarize. An integrated method of Top-down mainly by manual and Bottom-up mainly by computer is adopted to construct Ontology model.

\subsection{Domain Ontology}

The domain Ontology of CG design \& production process is denoted by a five-element model OCG $=\{C, R, P, A, I\}$. C is the set containing all the concepts related in CG design \& production process. Some main concepts and their hierarchical level in $\mathrm{C}$ is shown in Figure 3 (a). $\mathrm{R}$ represents the relationships among these concepts, containing parent-child 
relationship, whole-part relationship, object-attribute relationship and so on. The relationship is-a shown in Figure 3 (a) is a kind of parent-child relationship. As shown in Figure 3 (b), the relationship limitedBy means that CG design \& production process is limited by CG animation laws. The relationship use means that CG design \& production process will use some software, such as 3D modeling software, rendering software, texturing software and so on. The relationship use means that CG design \& production process could produce CG animation. Besides, there are reciprocal relationships, transfer relationships, function relationships and symmetrical relationships. For example the relationship limitedBy and limited are reciprocal.

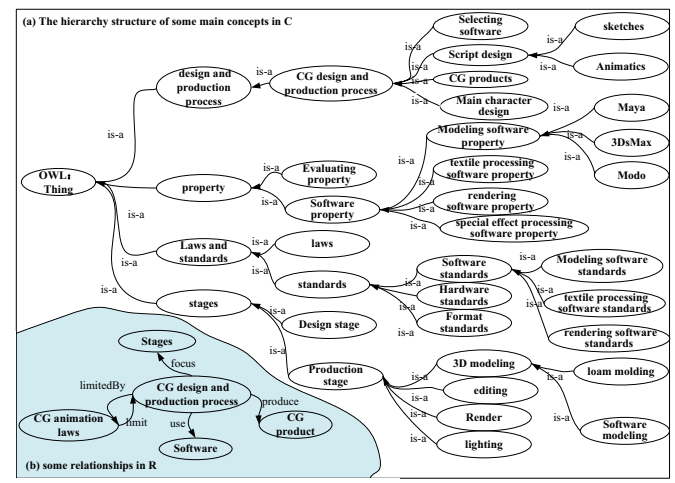

Figure 4. The domain Ontology of CG design \& production process (part)

$\mathbf{P}$ represents the set of properties, used in depicting the concept. $\mathbf{A}$ is the Axioms defined on $\sum \mathrm{O}_{\mathrm{CG}}\left(\right.$ where $\sum \mathrm{O}_{\mathrm{CG}}=\mathbf{C}$ $\cup \mathbf{R})$ depicting all the rules within this field. Axiom could put constraints on other concepts and properties. Take the concept software rule for example, it could be defined by the natural language as 'the software rule is used to select software'. This definition can be decomposed into two: (1) a software rule is a rule, and (2) this rule is used to select software. This concept could be expressed as follow axiom:

software rule $=$ rule $\cap(\forall$ rule. software selecting $)$

I represents the set1 of all the instances of $\mathbf{C}$.

\subsection{Application Ontology 1- domain knowledge}

The application Ontology for domain knowledge is shown in Figure 5. Some main concepts and the hierarchy relationship among them are shown in Figure 5 (a). The Document_Feature Ontology represents the features of document in CG design \& production process. According to their format, Document_Feature Ontology could be classified into several sub Ontology, such as usage feature, hierarchy feature, format feature, process feature, resource feature and so on. The Document Ontology represents knowledge carrier such as pictures, papers and so on. According to the classification of Document_Feature Ontology, document could be divided into corresponding classes. With the thining of document feature, document types are thinned further, and thus the hierarchy structure is formed just as shown in Figure 5 (a). The Ontology knowledge_Element could be divided into concept knowledge element, principle knowledge element, method knowledge element, fact knowledge element and so on. The properties of domain knowledge Ontology are shown in Figure 5 (b). The property hasFeature represents the features of domain knowledge. 


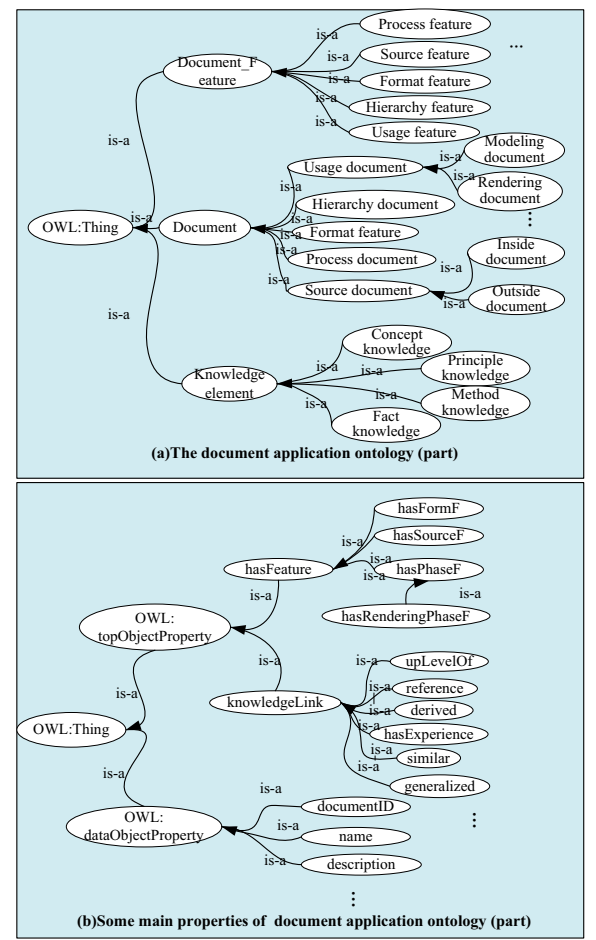

Figure 5. The application Ontology for domain knowledge (part)

\subsection{Application Ontology 2- process knowledge}

The process knowledge is the integration of all the interactive activities performed during the CG design \& production process. The main ontologies are shown in Figure 6. The Ontology activity represents all the activities accord in the CG design \& production process, which could be refined into publicActivity and privateActivity. The Ontology publicActivity signifies the activities occurred on the level of organizations, containing taskPlanning, taskDecomposing, taskRealizing, taskResultReducing. requirementAnalysis is the sub-class of taskPlanning. The Ontology privateActivity signifies the activities occurred on the level of individuals, containing designProcessActivity and designRationalActivity. designRationalActivity contains four thinking activities: convergent thinking activities, generative thinking activities, analytical thinking activities and synthesis thinking activities.

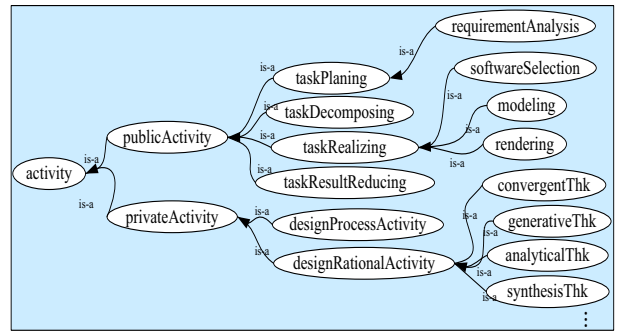

Figure 6. The process application Ontology (part) 


\section{Case study}

This case is a naked-eye 3D advertisement for hand sanitizers, which comes from an IT company located in Shanghai. The naked-eye 3D film is an emerging CG product, which is regarded as the next generation of display technology with endless vitality. To get the best naked-eye 3D visual effect, design \& production knowledge of each step should be reused and shared. Thus the proposed method was applied in the knowledge modeling of naked-eye 3D animation design and production to illustrate effectiveness of this method.

The procedures of naked-eye 3D film design \& production include the following key steps: script design, 3D modeling, scene making, rendering, film production, synthesis, editing etc. The knowledge classification of naked-eye 3D animation design \& production is shown in the lower part of Figure 3. Each kind of knowledge was modeling with Ontology technique. The activity of defining tasks could form links among the domain knowledge, design requirements (static knowledge) and the related tasks (dynamic knowledge). Thus the Ontology representation of the defining tasks activity is described in Figure 7 with OWL. Figure 7 (a) shows the integrated Ontology for defining tasks activity. The input and output of defining tasks activity Ontology are design target Ontology (Figure 7 (b)) and statement of task Ontology (Figure 7 (c)), respectively. The design target Ontology describes customer requirements in respects of pictures, voice, lens and so on. The statement of task Ontology is the formal statements of design task which are described in designer's language. During the defining tasks activity, domain knowledge Ontology (Figure 7 (d)) will be used. The final notes about Figure 7 are: "SPE****", such as "SPE2940", "SPE7293" etc., refers to relevant provisions. "TAB2098" refers to the task set. "RQT****", such as "RQT0712", "RQT0932" etc., refers to the design requirements of this design task. "YFSD2344" refer to the knowledge browsed in the process of task formulation.

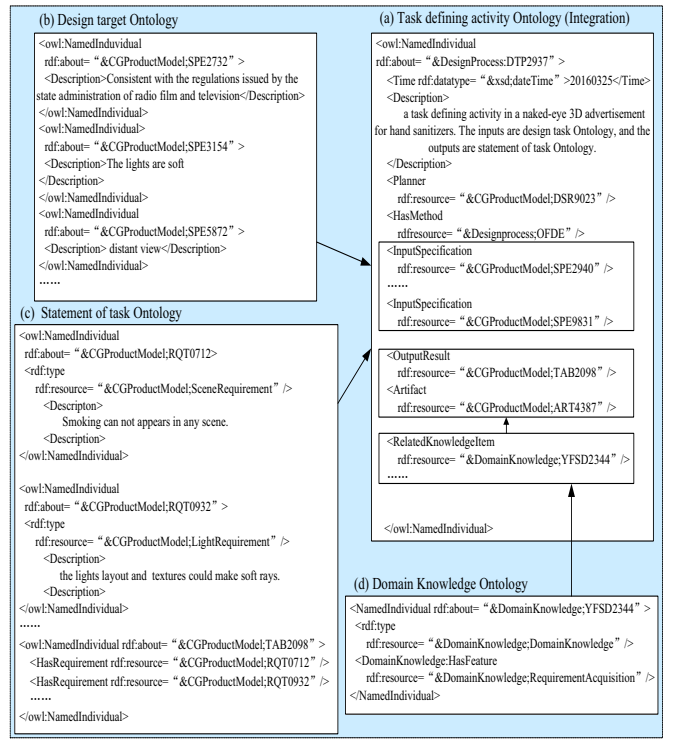

Figure 7. The OWL of the naked eye 3D film design \& production Ontology (part)

\section{Conclusion}

This article proposed an integrating knowledge modelling method to aid CG design \& production. CG animation knowledge is capture and organized from viewpoint of three aspects: requirements and design and production. The knowledge are categorized into static and dynamic knowledge, and Ontology is adopted to construct a hierarchic model to organize the knowledge, so as to offer a uniform communication semantic foundations for designers from different fields. 
Based on animation script, the CG design task model is proposed to drive the organization and management of different knowledge involved in CG design \& production.

\section{Acknowledgements}

This research was financially supported by National Natural Science Foundation of China (No.51405281) and Shanghai Science and Technology Innovation Action Plan (No.14511108303). The authors express sincere appreciation to the anonymous referees for their helpful comments to improve the quality of the paper.

\section{References}

1. Jin shen. Modelling and configuration of product extension services using an Ontology-based approach. Shanghai Jiao Tong University. 2012, Shanghai.

2. Gruber, T.R. A translation approach to portable ontology specifications. Knowledge Acquisition, 1993, 5(2): 199-220.

3. Jianjun Chen, Chenghu Zhou, Jinggui Wang. The research on geography Ontology. Geography frontier, 2006(03): 81-90.

4. FIORENTINI X, GAMBINO I, LIANG V, et al. An ontology for assembly representation. New York: Department of Commerce, 2007.

5. RAPHAEL B, KRIMA S, RACHURI S, et al. OntoSTEP : Enriching product model data using ontologies. Computer-Aided Design, 2012, 44(6): 575-590.

6. FIORENTINI X, GAMBINO I, LIANG V, et al. An ontology for assembly representation. New York: Department of Commerce, 2007.

7. MINNA L, GARCIA F, KALLELA T, et al. Product-process ontology for managing assembly specific knowledge between product design and assembly system simulation// Micro-Assembly Technologies and Applications. IFIP-International Federation for Information Processing 260. Springer US, 2008:99-108.

8. Weiwei Wang, Zhigang Wang, Liangming Pan, et al. Research on the construction of bilingual movie knowledge graph. Acta Scientiarum Naturalium Universitatis Pekinensis, 2016, 52 (1): 25-34.

9. Honggen Zhou, Mengying Wang, Xuwen Jing, et al. Modeling and application of diesel ontology model for multidisciplinary optimization. Computer Integrated Manufacturing Systems. 2014, 20 (10): 2348-2359.

10. Ma, S., Tian, L.Ontology-based semantic retrieval for mechanical design knowledge, International Journal of Computer Integrated Manufacturing, 2015, 28 (2):226-238.

11. Chen, Y., Zhao, M., Xie, Y., Zhang, Z. A new model of conceptual design based on Scientific Ontology and intentionality theory. Part II: The process model. (2015) Design Studies, 38, pp. 139-160. 